\title{
A Fast Method of Generating Pharmacokinetic Maps from Dynamic Contrast-Enhanced Images of the Breast
}

\author{
Anne L. Martel \\ Department of Medical Biophysics, University of Toronto, Canada \\ anne.martelesri.utoronto.ca
}

\begin{abstract}
A new approach to fitting pharmacokinetic models to DCE-MRI data is described. The method relies on fitting individual concentration curves to a small set of basis functions and then making use of a look up table to relate the fitting coefficients to pre-calculated pharmacokinetic parameters. This is significantly faster than traditional non-linear fitting methods. Using simulated data and assuming a Tofts model, the accuracy of this direct approach is compared to the Levenberg-Marquardt algorithm. The effect of signal to noise ratio and the number of basis functions used on the accuracy is investigated. The basis fitting approach is slightly less accurate than the traditional non-linear least squares approach but the ten-fold improvement in speed makes the new technique useful as it can be used to generate pharmacokinetic maps in a clinically acceptable timeframe.
\end{abstract}

\section{Introduction}

MRI has been shown to be a powerful technique for the diagnosis and assessment of breast cancer. The use of dynamic contrast-enhanced MRI (DCE-MRI) as a screening modality is currently under investigation in several centres [1,2], but although the negative predictive value of the technique approaches $100 \%$, which is desirable for a screening technique, the positive predictive value is much lower than for conventional mammography $[1,3]$. This indicates that many women with benign lesions are being referred for breast biopsies and much effort is currently being made to improve the PPV and specificity of MR breast imaging.

The MR images contain a great deal of information and once morphological features and parameters characterizing the contrast kinetics have been extracted from the data a computer classification scheme can be used to distinguish between malignant and benign lesions. Many investigators use simple methods to characterize the signal intensity versus time curves; a subjective scoring system which attempts to distinguish between curves with and without a wash-out phase has been described [4] and quantitative measures of uptake and wash-out ratios may also be used [5-7]. The main difficulty with these approaches is that the kinetic features extracted from the image data tend to vary between institutions due to differences in imaging equipment, protocol and analysis techniques.

In principle pharmacokinetic modeling is less sensitive to variations in MRI acquisition protocols and it has the additional advantage that parameters obtained in this manner have some underlying physiological meaning. The signal intensity versus 
time curves are first converted to concentration time curves and are then fitted to a compartmental model such as the Brix model [8] or the Tofts model [9]. Fitting the concentration time curves to a model is usually achieved using a non-linear least squares method algorithm such as the Levenberg-Marquardt method [10]. Concentration curves generated using a region of interest have a good signal to noise ratio due to averaging over many pixels and the time taken to carry out a fit of a single curve is not significant.

Generating parametric images where every pixel concentration curve must be fitted to a compartmental model is more problematic, however. Fitting techniques are sensitive to the starting values used and may fail to converge [11, 12], and the time taken to carry out the fit on every single pixel in a 3D volume image becomes a significant obstacle to the translation of pharmacokinetic modeling into clinical practice. This means that although pharmacokinetic modeling of DCE-MRI data has the potential to improve the diagnostic accuracy of screening studies it has not been used as extensively as less reproducible approaches such as mapping the percentage contrast enhancement or wash-out.

For this reason we have developed a new method of generating pharmacokinetic maps using basis functions. This technique will allow us to assess the utility of pharmacokinetic modeling for breast screening in future studies.

\section{Theory and Methods}

\subsection{Pharmacokinetic Modeling in DCE-MRI}

The compartmental model described by Tofts [9] is frequently used to characterize contrast dynamics in tumours. The concentration of Gd-DTPA contrast agent in the tumour is given by the expression

$$
C(t)=D K^{\text {trans }} \sum_{i=1}^{2} a_{i}\left(\frac{\exp \left(-k_{e p} t\right)-\exp \left(m_{i} t\right)}{m_{i}-k_{e p}}\right)
$$

where $C(t)$ is the tissue concentration and $D$ is the injected dose $(\mathrm{mmol} / \mathrm{kg})$. Published values were used for the rate constants of Gd-DTPA clearance $\left(m_{1}\right.$ and $\left.m_{2}\right)$ and for the corresponding amplitudes $\left(a_{1}\right.$ and $\left.a_{2}\right)$ [9]. Assuming that permeability-limited conditions apply, $K^{\text {trans }}\left(\mathrm{min}^{-1}\right)$ equals the transfer constant between the blood plasma and tissue compartments and $k_{e p}$ is equal to $K^{\text {trans }} / v_{e}$ where $v_{e}$ is the extracellular extravascular volume fraction.

Conventionally the parameters $K^{\text {trans }}$ and $v_{e}$ are determined using a non-linear least squares technique such as the Levenberg-Marquardt algorithm [10].

\subsection{Using Basis Functions to Estimate Pharmacokinetic Parameters}

Any concentration versus time curve can be represented by a linear combination of basis functions where

$$
C(t)=\sum_{k=1}^{m} a_{k} \phi_{k}(t)+e(t)
$$


$a_{k}$ are the fitting coefficients, $\phi_{k}(t)$ is the k'th basis function and $e(t)$ is an error term. Ideally a small number of basis functions should be sufficient to give a good approximation to $\mathrm{C}(\mathrm{t})$ and the basis functions themselves should have features matching those known to belong to the functions being estimated. Representing $\mathrm{C}(\mathrm{t})$ as a linear combination of basis functions has two advantages; firstly it smooths the curve and secondly if the number of basis functions required is small then the fitting coefficients provide a very efficient way of parameterising the curve. Commonly used methods of defining basis functions include Fourier series expansions and spline functions however these are generic basis functions and may not be as efficient at representing the signal intensity curves as a tailored set of basis functions would be. We propose to use principal components analysis (PCA) to generate a set of basis functions that are optimized to represent DCE-MRI signal intensity curves from breast studies.

First a population of "plausible" curves is generated using equation 1 . This was done by varying the values of $\mathrm{K}^{\text {trans }}\left(0.001<\mathrm{K}^{\text {trans }}<1.0, \Delta \mathrm{K}^{\text {trans }}=0.001 \mathrm{~min}^{-1}\right)$ and $\mathrm{v}_{\mathrm{e}}$ $\left(0.001<\mathrm{v}_{\mathrm{e}}<1.0, \Delta \mathrm{v}_{\mathrm{e}}=0.01\right)$ to give 20000 signal intensity curves. These curves are arranged into an $\mathrm{n} x \mathrm{t}$ matrix $\mathbf{X}$ where $\mathrm{n}=20000$ and $\mathrm{t}$ depends on the temporal resolution and the duration of the study. This matrix can be decomposed using singular value decomposition to give

$$
\mathbf{X}=\mathbf{U} \mathbf{W} \mathbf{V}^{\mathbf{T}}
$$

where $\mathbf{V}$ is an orthonormal ( $\mathrm{p} \times \mathrm{p}$ ) matrix, $\mathbf{W}$ is a diagonal ( $\mathrm{p} \times \mathrm{p}$ ) matrix where the diagonal elements are known as singular values and $\mathbf{U}$ is a column-orthonormal ( $\mathrm{n} x$ p) matrix. If the diagonal elements of $\mathbf{W}$ are sorted into descending order and the columns of $\mathbf{U}$ and $\mathbf{V}$ are reordered accordingly then equation (2) is equivalent to a PCA where the columns of $\mathbf{V}$ contain the principal component curves, the diagonal elements of $\mathbf{W}$ are equal to the square roots of the eigenvalues and the columns of $\mathbf{U}$ correspond to the coefficients. The first 6 principal components are shown in figure 1 . The first $\mathrm{m}$ PCs are used as basis functions where the selection of $\mathrm{m}$ is discussed later in this paper. The coefficients in the matrix $\mathbf{U}$ together with the corresponding values for $\mathrm{k}_{21}$ and $\mathrm{k}_{\mathrm{el}}$ are saved to create a lookup table. Any concentration curve $C(t)$ can then be expressed as a linear combination of the first $\mathrm{M}$ principal components

$$
C(t) \approx \sum_{k=1}^{m} a_{k} \phi_{k}(t)=\sum_{k=1}^{m} a_{k} w_{k k} \mathbf{v}_{k}(t)
$$

Or in matrix terms

$$
C=\mathbf{A} \Phi^{T}=\mathbf{A} \mathbf{W}^{\prime} \mathbf{V}^{T}
$$

where $\mathbf{C}$ is the ( $\mathrm{n} \times \mathrm{p}$ ) matrix with the rows representing the concentration curves for the $\mathrm{n}$ pixels, $\mathbf{A}$ is the ( $\mathrm{n} \times \mathrm{m}$ ) matrix of fitting coefficients, $\mathbf{W}^{2}$ is the ( $\mathrm{m} \mathrm{x} \mathrm{m}$ ) diagonal matrix containing the first $\mathrm{m}$ eigenvalues and $\mathbf{V}$ ' is the $(\mathrm{p} \times \mathrm{m})$ matrix containing the first $\mathrm{m}$ principal components. The fitting coefficients $\mathbf{A}$ are estimated by

$$
\mathbf{A}=\mathbf{C} \boldsymbol{\Phi}\left(\boldsymbol{\Phi}^{T} \boldsymbol{\Phi}\right)^{-1}
$$

The coefficients corresponding to the $\mathrm{j}$ 'th pixel SI curve are then compared against those generated for the reference curves to find the closest point. 


$$
i(j)=\min _{i} \sum_{k=1}^{k}\left(a_{j k}-u_{i k}\right)^{2}
$$

Parametric images of $\mathrm{K}^{\text {trans }}$ and $\mathrm{v}_{\mathrm{e}}$ are then generated by looking up the values corresponding to the ith reference curve.
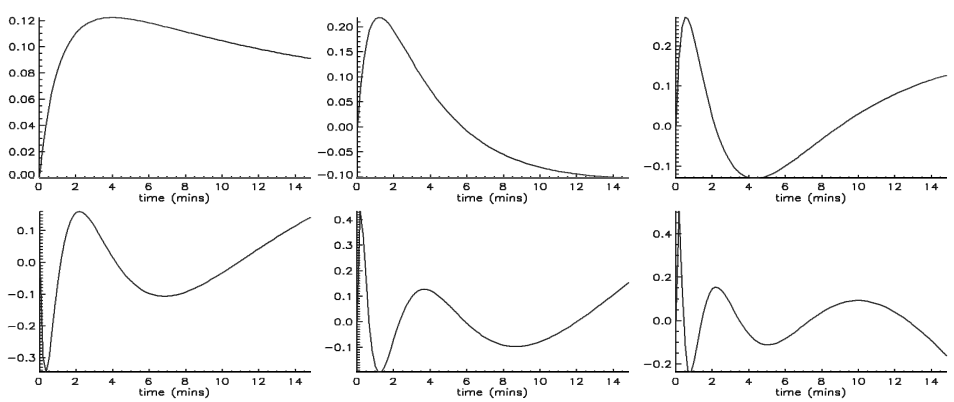

Fig. 1. The first 6 basis functions

\subsection{Simulation Studies}

In order to test the accuracy of this approach and to determine how many basis functions were required, a test set of 55 curves was generated with combinations of $\mathrm{K}^{\text {trans }}$ and $\mathrm{v}_{\mathrm{e}}$ values selected over the same range as that used to generate the reference curves. A temporal resolution of 10 seconds and scan duration of 15 minutes was assumed. The parameter estimates obtained using a Levenburg-Marquardt algorithm [11] were compared with results obtained using the basis function approach outlined above. The following conditions were varied

- In addition to the noise free case, Gaussian noise was added to each curve to give signal to noise ratios of 10 and 20 (SNR defined as peak concentration/noise SD). 1000 noisy curves were generated for each of the $55 \mathrm{~K}^{\text {trans }}$ and $\mathrm{v}_{\mathrm{e}}$ combinations to allow median values and 5\%-95\% confidence intervals to be calculated.

- The number of basis functions used was varied between 2 and 6

\subsection{Patient Study}

The proposed technique was tested using a DCE-MRI study carried out as part of an on-going screening study. During the first 2 minutes postinjection, 11 dynamic images were acquired using a 2-dimensional SPGR sequence with fat saturation (TR/TE/flip angle, $150 \mathrm{~ms} / 4.2 \mathrm{~ms} / 50^{\circ}$ ) and a temporal resolution of 20 seconds. This was followed by a single high spatial resolution 3-dimensional SPGR scan that took 7 minutes and was used to provide morphological information. This was then followed up by an additional series of 3 dynamic images to monitor contrast media washout. A contrast dose of $0.1 \mathrm{mmol} / \mathrm{kg}$ Gd-DTPA was injected after the third image was acquired. Each image comprised 256 × 256 pixel and there were 12 slices. A uniform 
T1 value of $710 \mathrm{~ms}$ was assumed in order to convert the signal intensity values into concentration values. Pharmacokinetic parameters were then calculated from the concentration data using the non-linear least squares technique (NLLS) described in [11] and using the basis function fitting method described above with $\mathrm{m}=2$.

\section{Results}

In Fig. 2 the estimated values of $\mathrm{K}^{\text {trans }}$ obtained using noise free simulation data are plotted against the corresponding value of $\mathrm{v}_{\mathrm{e}}$ and the true values of $\mathrm{K}^{\text {trans }}$ are given on the right. Concentration curves corresponding to small $\mathrm{v}_{\mathrm{e}}$ and high $\mathrm{K}^{\text {trans }}$ show very rapid early increase followed by a rapid washout and it is clear from the results shown in figure 2 that the basis functions do not fit the data well in this situation. The errors in the estimated $\mathrm{K}^{\text {trans }}$ values increase as the number of basis functions used decreases.
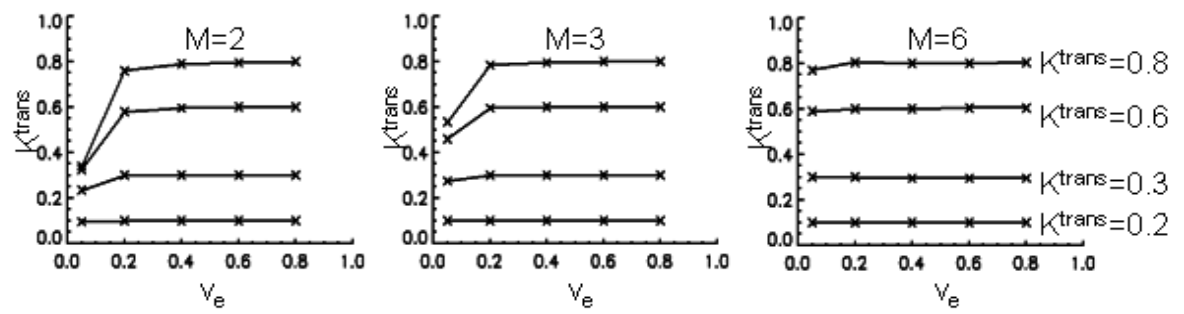

Fig. 2. Effect of altering $m$, the number of basis functions used on noise free data
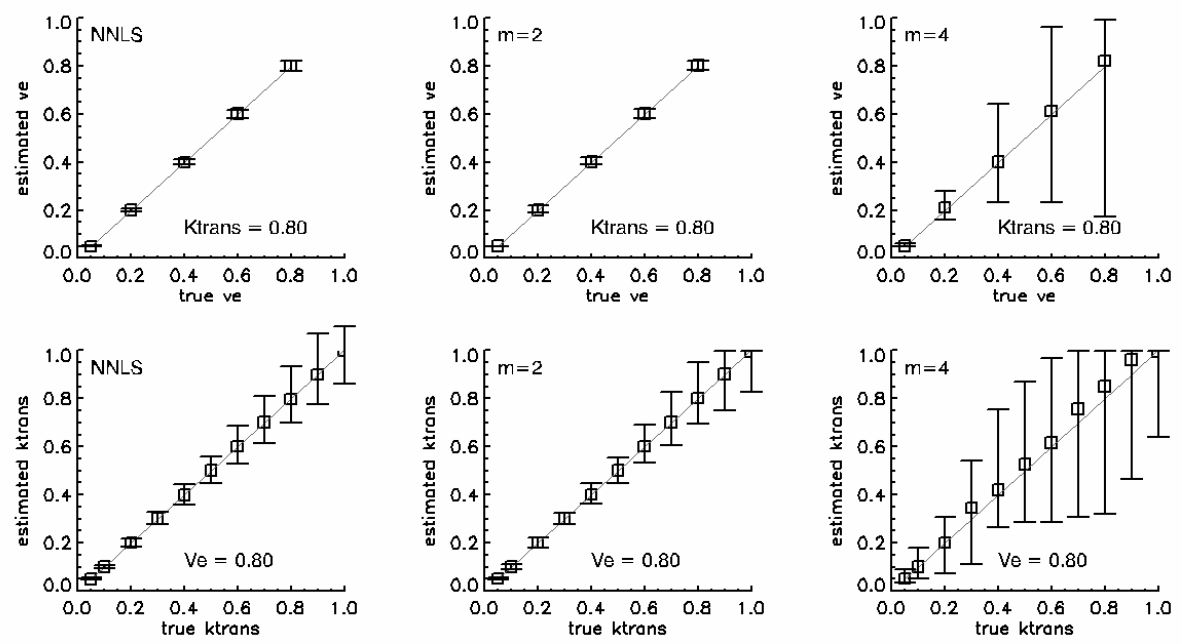

Fig. 3. Median values and 5\%-95\% confidence intervals are plotted for a subset of the simulation curves $(\mathrm{SNR}=10)$. $\mathrm{K}^{\text {trans }}$ measurements for $\mathrm{v}_{\mathrm{e}}=80 \%$ (top row), and $\mathrm{v}_{\mathrm{e}}$ measurements for $\mathrm{K}^{\text {trans }}=0.8$ (bottom row). When $\mathrm{SNR}=10$, overfitting occurs for $\mathrm{m}>3$. 

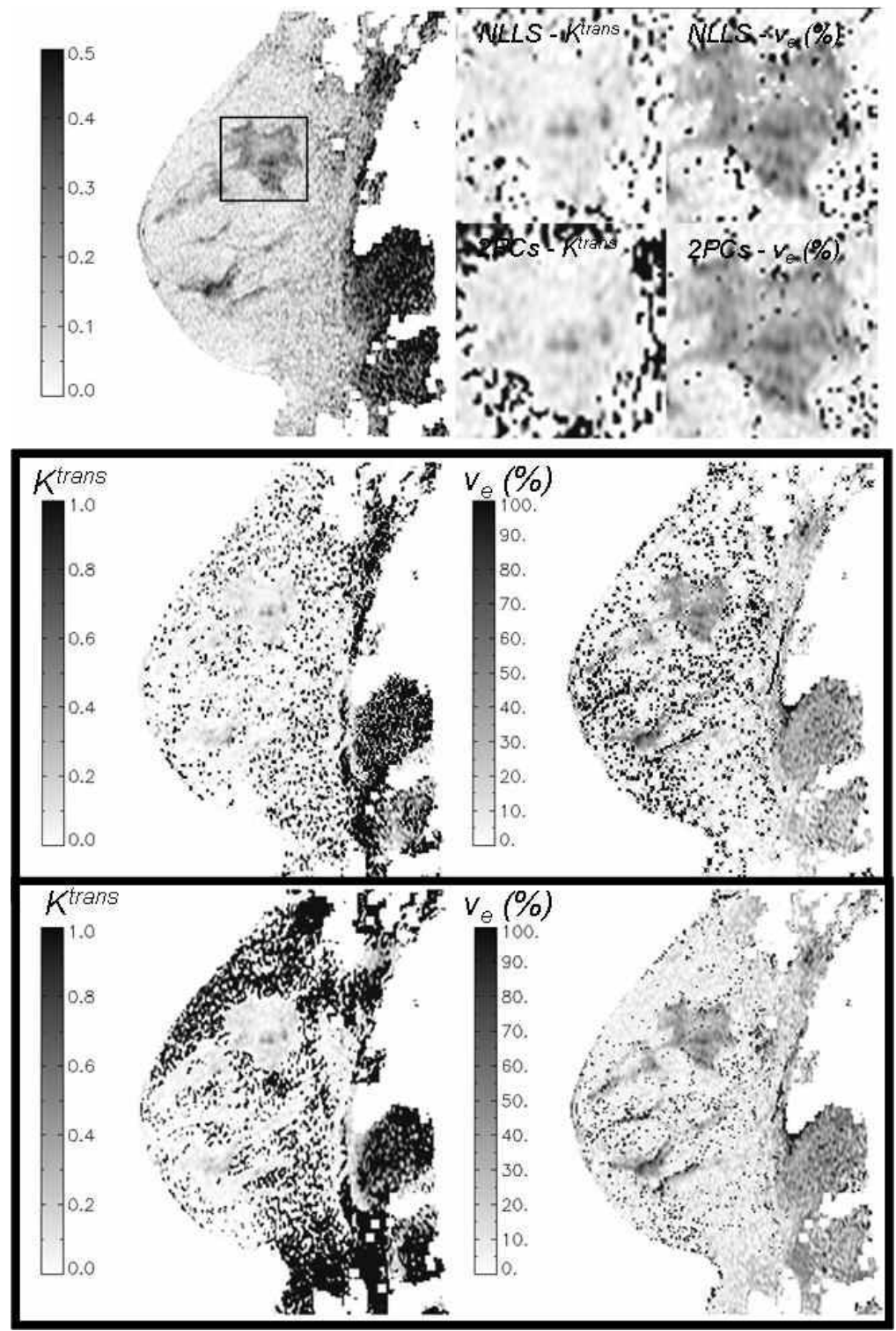

Fig. 4. (Upper left) Slice taken from a DCE-MRI study of a patient with a fibroadenoma in the left breast. (Middle row) Pharmacokinetic maps generated using a conventional NLLS technique. (Lower row) Maps generated using 2 basis functions. (Upper right) Close-up view of region defined by ROI showing excellent agreement between methods.

The results from the noise free simulations suggest that a higher number of basis functions is desirable but in noisy data there is a tradeoff between bias in the fitted curve and variance in the fitting coefficients. In the noisy simulations, results suggest 
that over-fitting occurs when $m>4$ for $\mathrm{SNR}=20$ and $\mathrm{m}>3$ for $\mathrm{SNR}=10$ and we found that good results could be obtained using $\mathrm{m}=2$ or 3 . Provided that $\mathrm{v}_{\mathrm{e}}>20 \%$ there was no significant reduction in accuracy. Increased error in the $\mathrm{K}^{\text {trans }}$ values due to bias were seen in curves where $\mathrm{v}_{\mathrm{e}}<20 \%$. Fig. 3 shows a subset of the simulation results for $\mathrm{SNR}=20$.

In Fig. 4 it can be seen that there is excellent agreement between the maps obtained using a conventional NNLS approach and the basis fitting approach where $m=2$. The time taken to carry out the Levenberg-Marquardt fitting on a $3 \mathrm{GHz}$ Xeon processor was 1 hour, 26 minutes compared to 9 minutes for the basis function approach.

\section{Discussion and Conclusions}

We have reduced the processing time required to generate pharmacokinetic parameter maps from pixel concentration curves by a factor of 10. This means that images showing pharmacokinetic parameters can be more readily utilised in a clinical screening environment where lengthy pre-processing of image data may not be viable. The faster technique has introduced some errors in the calculated parameters for curves generated with low values of $\mathrm{v}_{\mathrm{e}}$ and further work is needed to determine whether this affects diagnostic accuracy. An adaptive technique that allows more basis curves to be used for pixels with a greater SNR may reduce the error.

Although this paper has described a method to fit a Tofts model to the concentration data it is possible to use any pharmacokinetic model provided that the "model space" of possible concentration curves can be defined a priori. We have also fitted data to a modified Brix model [12] using this approach with similar results (unpublished data).

Another application of the technique is as a pre-processing step for conventional non-linear least-squares routines where accuracy is important. The pharmacokinetic parameters obtained using the basis fitting technique could be used as initial starting estimates for the Levenberg-Marquardt technique which is very sensitive to the choice of initial values.

\section{Acknowledgements}

This work was supported by the Canadian Breast Cancer Research Alliance. We would like to thank Don Plewes for providing the breast MRI data.

\section{References}

1. Warner, E., et al., Surveillance of BRCA1 and BRCA2 mutation carriers with magnetic resonance imaging, ultrasound, mammography, and clinical breast examination. JAMA, (2004). 292(11): 1317-25.

2. Brown, J., et al., Magnetic resonance imaging screening in women at genetic risk of breast cancer: imaging and analysis protocol for the UK multicentre study. Magnetic Resonance Imaging, 2000. 18(7): 765-776. 
3. Warren, R.M., et al., Reading protocol for dynamic contrast-enhanced MR images of the breast: sensitivity and specificity analysis. Radiology, 2005. 236(3): 779-88.

4. Kuhl, C.K., et al., Dynamic breast MR imaging: are signal intensity time course data useful for differential diagnosis of enhancing lesions? Radiology, 1999. 211: 101-10.

5. Heywang, S.H., et al., MR imaging of the breast with Gd-DTPA: use and limitations. Radiology, 1989. 171(1): 95-103.

6. Kaiser, W.A. and E. Zeitler, MR imaging of the breast: fast imaging sequences with and without Gd-DTPA. Preliminary observations. Radiology, 1989. 170: 681-6.

7. Gibbs, P., et al., Differentiation of benign and malignant sub-1 $\mathrm{cm}$ breast lesions using dynamic contrast enhanced MRI. Breast, 2004. 13(2): 115-21.

8. Brix, G., et al., Pharmacokinetic parameters in CNS Gd-DTPA enhanced MR imaging. J Comput Assist Tomogr, 1991. 15(4): 621-8.

9. Tofts, P.S., B. Berkowitz, and M.D. Schnall, Quantitative analysis of dynamic Gd-DTPA enhancement in breast tumors using a permeability model. Magn Reson Med, 1995. 33: 564-8.

10. Press, W.H., et al., Numerical Recipes in C. 1994, Cambridge: Cambridge University Press.

11. Ahearn, T.S., et al., The use of the Levenberg-Marquardt curve-fitting algorithm in pharmacokinetic modelling of DCE-MRI data. Phys Med Biol, 2005. 50(9): p. N85-92.

12. Buckley, D.L., et al., Quantitative analysis of multi-slice Gd-DTPA enhanced dynamic MR images using an automated simplex minimization procedure. Magn Reson Med, 1994. 32(5): 646-51. 